\title{
REVIEW
}

\section{Melanocytic dysplastic naevi occupy the middle ground between benign melanocytic naevi and cutaneous malignant melanomas: emerging clues}

\section{R Hussein}

J Clin Pathol 2005;58:453-456. doi: 10.1136/icp.2004.019422

Although several studies have confirmed the aetiological importance of melanocytic dysplastic naevi (MDN) in the development of cutaneous malignant melanoma (CMM), the analysis of these lesions was directed mostly towards the study of melanomas. The underlying reasons include the relatively large size of $\mathrm{CMMs}$, their direct lethal outcome, and the feasibility of establishing melanoma cell lines. In contrast, because of their relatively small size, questionable malignant potential, and the difficulty in establishing in vitro cultures, MDN have been studied less extensively. Hypothetically, transformed melanocytes can give rise to any lesion in the hierarchy of melanocytic tumours. Based on this hypothetical perspective, and on the epidemiological, morphological, immunohistochemical, and genetic characteristics of MDN, it is not surprising that these lesions occupy an intermediate position in the hierarchy of melanocytic lesions, and may be precursors of CMM. Although this argument appears to be straightforward, it is still controversial. This review explores the components of this argument and provides supporting evidence for this hypothesis.

Correspondence to:

Dr M R Hussein,

Department of Pathology, Assiut University Hospitals, Assiut, Egypt; mrh17@ swissinfo.org

Accepted for publication 18 July 2004
A lthough melanocytic dysplastic naevi $(\mathrm{MDN})$ are aetiologically important in the development of cutaneous malignant melanoma (CMM), these lesions have not been studied extensively. Hypothetically, transformed melanocytes can give rise to any lesion in the hierarchy of melanocytic tumours. Based on this hypothesis, and on the epidemiological, morphological, immunohistochemical, and genetic characteristics of MDN, it is not surprising that these lesions occupy an intermediate position in the hierarchy of melanocytic lesions, and may be precursors of CMM. Although this argument appears to be straightforward, it is still controversial. This review will explore the components of this argument and provide supporting evidence for this hypothesis.

\section{INTRODUCTION}

Historical aspects and incidence of CMM The term "black cancer" was first used to describe CMM by Hippocrates in the fifth century BC. In 1806, René Laennec provided the first description of melanoma as a disease entity, and his manuscript of 1812 , reporting a case of disseminated melanoma, also marks the first published use of the word melanoma. ${ }^{1}$

The incidence of melanoma has increased dramatically worldwide, posing a major threat to public health. For example, in the USA the incidence of melanoma is rising faster than that of any other cancer, with an estimated 20000 to 40000 cases of in situ CMM and 47700 cases of invasive CMM diagnosed each year. ${ }^{2}$

\section{Historical aspects of MDN}

Although the concept of MDN was introduced only recently, these lesions were observed a long time ago. The observation was made in the context of melanoma in 1820 by William Norris, who arrived at the conclusion that melanoma is a hereditary disease associated with the development of moles. In 1829, Norris $^{3}$ described a family in which two members developed melanomas and the other family members had many moles on various parts of their bodies. In 1974, Munro $^{4}$ described macroscopic and microscopic pictures of what is now known as MDN. In 1978, Clark et al published the first report to advance MDN as a separate pathological entity associated with an increased risk of melanoma. The lesions were reported in two familial melanoma kindreds with multiple moles, and were named after the initials of the index families under the new designation of B-K mole. ${ }^{5}$ Later, they were referred to as large atypical naevi. In 1983, Lynch et al described a similar kindred and introduced the term "familial atypical multiple mole melanoma syndrome". ${ }^{\circ}$ In 1980, Greene and his colleagues applied the term dysplastic naevi, because the lesions have clinical, architectural, and cytological atypical features. ${ }^{7}$

\section{MDN and CMM}

Although the exact cause of melanoma is unknown, numerous studies defined the presence of MDN as an important factor associated with an increased risk of melanoma. These dysplastic lesions are clinically different from common acquired naevi (table 1). Some patients may have many MDN and are at increased risk for the development of melanoma; a condition collectively known as the dysplastic naevus syndrome. ${ }^{6}$

Abbreviations: $\mathrm{BN}$, benign naevi; $\mathrm{CMM}$, cutaneous malignant melanoma; MDN, melanocytic dysplastic naevi; UVR, ultraviolet radiation 
Table 1 Summary of the morphological, immunohistochemical, and molecular features of melanocytic skin lesions

\begin{tabular}{|c|c|c|c|c|}
\hline Feature & $\mathrm{BN}$ & MDN & Early CMM & Refs \\
\hline \multicolumn{5}{|l|}{ Clinical } \\
\hline Symmetry & Symmetrical & Asymmetrical & Asymmetrical & $5-8,9$ \\
\hline Size & Small $(<5 \mathrm{~mm})$ & Larger (>5 mm) & Variable & $5-8,9$ \\
\hline Border & Regular & Irregular & Irregular & $5-8,9$ \\
\hline Colour & Uniform & Variegated & Variegated & $5-8,9$ \\
\hline \multicolumn{5}{|l|}{ Histological } \\
\hline Cytological atypia & Absent & Mild to severe & Severe & $5-8,9$ \\
\hline Architectural atypia & Absent & Mild to severe & Severe & $5-8,9$ \\
\hline \multicolumn{5}{|l|}{ Ultrastructural } \\
\hline Melanosomes & Normal & Aberrant & Aberrant & 10,11 \\
\hline Golgi & Normal & Well developed & Well developed & 10,11 \\
\hline Mitochondria & Numerous & Abundant & Abundant & 10,11 \\
\hline \multicolumn{5}{|l|}{ Immunohistochemical } \\
\hline MMR protein expression & Strong & Intermediate & Decreased & 12 \\
\hline p53, HMB45 expression & Absent & Intermediate & Pronounced & 13,14 \\
\hline Proliferation marker expression & Low & Intermediate & High & $15-17$ \\
\hline \multicolumn{5}{|l|}{ Genetic } \\
\hline MSI & Absent/Present & Present $(+)$ & Present $(+++)$ & 18,19 \\
\hline Allelic loss & Absent & Present $(+)$ & Present $(+++)$ & $20-22$ \\
\hline Loss of TSG (p16 and p53) & Absent & Present $(+)$ & Present $(+++)$ & 23 \\
\hline Alterations of oncogenes (ras) & Absent & Present $(+)$ & Present $(+++)$ & 24 \\
\hline
\end{tabular}

BN, benign naevi; CMM, cutaneous malignant melanoma; $M D N$, melanocytic dysplastic naevi; MMR, mismatch repair; MSI, microsatellite instability; TSG, tumour suppressor gene.

\section{SCOPE OF THIS REVIEW}

Although many MDN are stable, some show atypical histological and cytological features, extending from mild to severe atypia that borders on CMM. Some groups have presented several examples of CMMs that have developed in pre-exiting $\mathrm{MDN}^{8}$ and consider MDN to be a precursor of CMM. Over the past few years, four lines of evidence have supported the concept of MDN as a major risk factor for, if not a direct precursor of, melanoma. These lines of evidence comprise the following: (1) epidemiological evidence, (2) morphological evidence, (3) immunohistochemical evidence, and (4) genetic evidence. Because available reviews about these issues are scarce, this review will analyse this evidence and concisely fill this gap in the literature.

\section{The epidemiological evidence}

MDN are relatively common lesions in the USA, where several million individuals have MDN but no family history of melanoma. In addition, it is estimated that approximately 32000 people have the familial dysplastic naevus syndrome, which is seen in association with familial melanoma. ${ }^{25}$ Four lines of evidence support the notion that MDN are a crucial marker for increased risk of CMM. First, MDN prevalence surveys performed in CMM studies found an increased risk of CMM in patients with $\mathrm{MDN}^{26-29}$ In this regard, the prevalence of MDN in the general population and in patients with sporadic CMM has been estimated to be approximately $5 \%$ and $15 \%$, respectively. Second, the presence of MDN is associated with almost $100 \%$ and $60 \%$ of cases of familial and sporadic CMM, respectively. ${ }^{30}{ }^{31}$ Third, the presence of MDN carries an eightfold or greater risk for malignant melanoma. ${ }^{30}$ For example, the presence of one MDN has been associated with a twofold risk, whereas 10 or more confer a 12 fold risk. ${ }^{32}$ Fourth, ultraviolet irradiation (UVR), an important aetiological factor in the development of CMM, seems to be involved in a similar way in the development of $\mathrm{MDN} .{ }^{33}$ In support of this, fibroblasts from patients with CMM/MDN are very sensitive to cell killing by UVR and the UVR mimetic carcinogen, 4-nitroquinoline-1-oxide. ${ }^{34}$ Moreover, shuttle vector studies have revealed that lymphoblastoid cell lines from patients with MDN have a reduced ability to handle UVR induced DNA damage, ${ }^{36}$ and skin fibroblasts from these patients show more chromatid breaks after $x$ ray irradiation.
Furthermore, both major and minor structural and numerical abnormalities, reflecting general chromosomal instability, were seen in patients with MDN. ${ }^{37}$

\section{Morphological evidence \\ Histological features}

Dysplastic naevi are considered to be naevi with variable mild architectural and cytological atypia to severe atypia bordering on melanoma in situ (fig 1; table 1). ${ }^{32}$ Dysplastic naevus cells are usually arranged along the basal cell layer or extending downwards into the upper dermis. Cell nests are confined to the thickened papillary dermis and are scattered at unequal distances from each other. Cytologically, dysplastic naevus cells have small oval nuclei, surrounded by abundant amounts of faintly stained cytoplasm. Five lines of histological evidences support the notion that MDN fill the gap between benign naevi (BN) and CMM. (1) Some of dysplastic naevus cells exhibit "random mild to severe cytological atypia" and mitotic figures similar to early melanomas. ${ }^{5}$ In this regard, these atypical naevus cells have a size 1.3 to 1.5 times larger than the reference melanocytes. Their nuclei vary from 8 to $24 \mu \mathrm{m}$ in diameter, they have relatively abundant cytoplasm, and contain hyperchromatic nuclei with occasional visible nucleoli and delicate chromatin strands. The nuclear membrane may be folded or deeply indented. (2) Early photographic documentation revealed the transformation of some of these lesions into melanoma. (3) The presence of residual MDN in some excised melanomas. ${ }^{9}$ (4) A quantitative light microscopic study with DOPA staining revealed that the number and mean diameter of melanocytes in MDN is similar to that seen in superficial spreading melanomas. ${ }^{10}$ (5) Some research groups have presented several examples of CMMs that developed in preexiting MDN. ${ }^{38}$

\section{Ultrastructural features}

Electron microscopic studies revealed that MDN with severe dysplasia share several features with radial growth phase melanomas, including: (1) the large size of the dysplastic naevus cells, (2) bizarre shaped and pleomorphic nuclei, (3) well developed Golgi, (4) abundant and deranged mitochondria, and (5) aberrant melanosomes with deranged structures and irregular melanisation. ${ }^{11}$ Moreover, the atypical intraepidermal melanocytes of MDN contain a much higher 

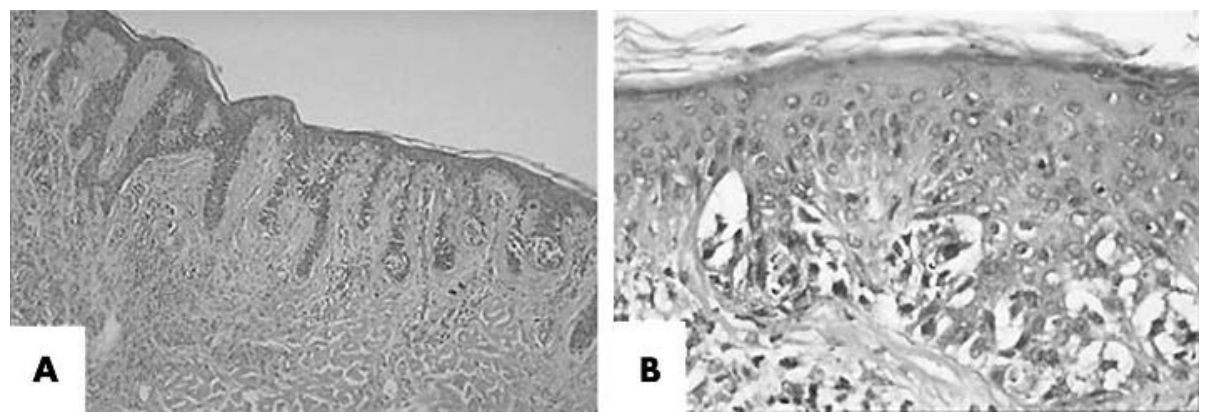

Figure 1 Histological appearance of a melanocytic dysplastic naevus (A, original magnification, $\times 100$; $\mathrm{B}$ original magnification, $\times 400$ ).

proportion of abnormal melanosomes than those in BN, similar to that seen in CMM. ${ }^{39}$ The complete absence of these features in $\mathrm{BN}$ and their presence in MDN emphasises the middle ground that MDN occupy among benign and malignant melanocytic lesions (table 1).

\section{Immunohistochemical evidence}

Several experimental observations support the notion that MDN represent intermediate lesional steps in a progression leading to CMM (table 1). ${ }^{12}$ These observations include: (1) proliferation marker (proliferating cell nuclear antigen/ cyclin) studies revealed that the reactivity of MDN is intermediate between $\mathrm{BN}$ and $\mathrm{CMM}^{15-17} ;(2)$ analysis of the expression patterns of mismatch repair proteins revealed that expression in MDN was intermediate between $\mathrm{BN}$ and $\mathrm{CMM}^{12}$; (3) the cell cycle protein p53 accumulates in $\mathrm{MDN}^{13}$ but at much lower overall frequency than in malignant melanoma ${ }^{40} ;(4)$ histocompatibility locus antigen class I abnormalities are similar in both CMM and dysplastic naevus cells $\mathrm{s}^{41}$; and (5) dysplastic naevus cells mildly to moderately express HMB45, a protein that is expressed by melanoma cells, but not by dermal naevus cells within BN. ${ }^{14}$

\section{Genetic evidence}

The finding of genetic changes in MDN similar to those found in CMM suggests that some of these dysplastic lesions are intermediate transitional stages in the course of melanoma tumorigenesis. ${ }^{12} 182021$ These changes include: (1) flow cytometry studies have found an increased frequency of abnormal DNA content in $\mathrm{MDN}^{42}$; (2) linkage analysis studies have suggested that CMM and MDN may be pleotropic manifestations of the same melanoma susceptibility genes at the $1 p$ and $9 p$ regions ${ }^{43}$; (3) the similar allelic losses at the $1 \mathrm{p}, 9 \mathrm{p}$, and $17 \mathrm{p}$ regions $\mathrm{s}^{20-23}$ in MDN and CMM support the existence of a biological continuum between some MDN and $\mathrm{CMM}^{44}$; (4) the same microsatellite instability (MSI) pattern is present in MDN at a frequency that is intermediate between $\mathrm{BN}$ and $\mathrm{CMM}^{18}{ }^{19}$; (5) TP53 and ras gene mutations occur in MDN at a much lower frequency than in melanomas; and (6) DNA abnormalities in MDN and superficial spreading melanomas are similar. ${ }^{24}$

\section{THE CLINICAL RELEVANCE OF DYSPLASTIC NAEVI}

The clinical relevance of MDN lies in their association with an increased risk of CMM. Cohort and case-control studies have shown that this increased risk occurs in several clinical settings. First, patients with numerous MDN are at a higher risk of developing CMM than those individuals with a single or only a few MDN. Second, patients with a personal or family history of CMM have an increased incidence of MDN. In this regard, there is a 100 fold increase in the incidence of MDN in patients who have previously had CMM, a 200 fold increase in those with at least two family members with CMM, and a more than 1200 fold increase in those with both a personal and a family history of melanoma. ${ }^{26-29} 45$

\section{THE HEATED CONTROVERSY ABOUT DYSPLASTIC NAEVI}

To date, the "dysplastic naevus" remains a controversial issue and contrary views about its existence are reported repeatedly in the literature. Part of the problem with these lesions is the lack of a precise definition. Some investigators such as Clark and co-workers considered MDN as: (1) lesions with aberrant melanocytic differentiation, and (2) premalignant lesions that can develop into CMM. Alternatively, other investigators such as Ackerman and co-workers considered MDN as: (1) wholly benign lesions, (2) variants of common acquired naevi, (3) the most common melanocytic naevus in humans, and (4) naevi of no clinical importance.

Ackerman proposed several reasons to explain why Clark and co-workers have been "led astray" about the issue of MDN. First, Clark and colleagues had the preconceived idea of the evolutionary nature of the lesions from benignancy (BN), to intermediacy (premalignant lesions), to malignancy (CMM). Accordingly, they thought that MDN would be the

\section{Take home messages}

- Several lines of evidence suggest that melanocytic dysplastic naevi (MDN) are aetiologically important in the development of cutaneous malignant melanoma (CMM)

- MDN are epidemiologically associated with the occurrence of CMM: there is an increased risk of CMM in patients with MDN; MDN are found in almost $100 \%$ and $60 \%$ of familial and sporadic CMMs, respectively; the presence of MDN carries an eightfold or greater risk for malignant melanoma; and ultraviolet irradiation is involved in the development of both MDN and CMM

- MDN share some morphological features with CMM and appear to fill the gap between benign naevi (BN) and CMM

- Immunohistochemistry has shown that the expression of proliferation markers, mismatch repair proteins, p53, and $\mathrm{HMB}-45$ is intermediate between $\mathrm{BN}$ and $\mathrm{CMM}$, and that both lesions show abnormalities in histocompatibility class I expression

- Molecular studies have revealed genetic aberrations in MDN that are intermediate between those found in BN and CMM, including abnormalities of DNA content, allelic losses, p53 and ras gene mutations, and microsatellite instability

- Thus, MDN appear to represent intermediates in the pathway of melanomagenesis and may be precursors of CMM, although this idea is still controversial, and more work is required to prove it 
intermediate lesional step in the hierarchy of melanocytic neoplasms. Second, what is now known about MDN, because Clark's naevi were biopsied only rarely in those days. Third, Clark and co-workers biopsied only large and unusual naevi in their patients with familial melanoma, but not smaller ones of more conventional appearance-that is, the appearance of BN. Therefore, they did not recognise that the histopathological features described as being typical of MDN were common to both MDN and BN. In addition, they failed to realise that the MDN were merely larger variants of $\mathrm{BN}$. Fourth, Clark and co-workers did not have consistent criteria for the recognition of MDN; therefore, they were carried away by speculations that supported their preconceived idea. For instance, the mutagenic effects of UVR in the initiation, promotion, and progression of melanocytic lesions..$^{5} 8384647$

\section{CONCLUSIONS}

Some MDN are not a "genetic dead end" but rather legitimate precursors of malignant melanoma. In this regard, MDN deserve this legitimacy because they almost fulfil the four requirements of precursor cells: (1) they are epidemiologically associated with the occurrence of CMM; (2) they share some morphological features with CMM; (3) they have immunohistochemical and (4) genetic characteristics that are intermediate between $\mathrm{BN}$ and CMM. Although current knowledge suggests that MDN do represent intermediates in the pathway of melanomagenesis, our current understanding of both the changes in MDN and the nature of these lesions is rudimentary, and much effort will be required to improve it.

Former address: Department of Dermatology, School of Medicine, University of Wisconsin, Madison, WI 53715, USA

\section{REFERENCES}

1 Weaver PC. The black cancer-malignant melanoma. Nurs Times 1976:72:582-4.

2 Greenlee RT, Murray T, Bolden S, et al. Cancer statistics, 2000. CA Cancer J Clin 2000;50:7-33.

3 Acharya S, Wilson T, Gradia S, et al. hMSH2 forms specific mispair-binding complexes with hMSH3 and hMSH6. Proc Natl Acad Sci U S A 1996;93:13629-34.

4 Munro DD. Multiple active junctional naevi with family history of malignant melanoma. Proc R Soc Med 1974;67:594-5.

5 Clark WH Jr, Reimer RR, Greene M, et al. Origin of familial malignant melanomas from heritable melanocytic lesions. "The B-K mole syndrome" Arch Dermatol 1978;114:732-8.

6 Lynch HT, Fusaro RM, Danes BS, et al. A review of hereditary malignant melanoma including biomarkers in familial atypical multiple mole melanoma syndrome. Cancer Genet Cytogenet 1983;8:325-58.

7 Greene $\mathbf{M H}$, Clark WH Jr, Tucker MA, et al. Precursor naevi in cutaneous malignant melanoma: a proposed nomenclature. Lancet 1980;2:1024.

8 Clark WH Jr. Tucker MA. Problems with lesions related to the development of malignant melanoma: common nevi, dysplastic nevi, malignant melanoma in situ, and radial growth phase malignant melanoma, Hum Pathol 1998;29:8-14.

9 Rhodes AR, Mihm MC Jr, Weinstock MA. Dysplastic melanocytic nevi: a reproducible histologic definition emphasizing cellular morphology [published erratum appears in Mod Pathol 1989;2:426] [see comments]. Mod Pathol 1989;2:306-19.

10 Rhodes AR, Melski JW, Sober AJ, et al. Increased intraepidermal melanocyte frequency and size in dysplastic melanocytic nevi and cutaneous melanoma. A comparative quantitative study of dysplastic melanocytic nevi, superficial spreading melanoma, nevocellular nevi, and solar lentigines. J Invest Dermatol 1983;80:452-9.

11 Langer K, Rappersberger K, Steiner A, et al. The ultrastructure of dysplastic naevi: comparison with superficial spreading melanoma and common naevocellular naevi. Arch Dermatol Res 1990;282:353-62.

12 Hussein MR, Roggero E, Sudilovsky EC, et al. Alterations of mismatch repair protein expression in benign melanocytic nevi, melanocytic dysplastic nevi, and cutaneous malignant melanomas. Am J Dermatopathol $2001 ; 23: 308-14$.

13 Lassam NJ, From L, Kahn HJ. Overexpression of p53 is a late event in the develop ment of malignant melanoma. Cancer Res 1993;53(10 suppl):2235-8.

14 Smoller BR, McNutt NS, Hsu A. HMB-45 staining of dysplastic nevi. Support for a spectrum of progression toward melanoma. Am J Surg Pathol 1989; 13:680-4.

15 Takahashi H, Strutton GM, Parsons PG. Determination of proliferating fractions in malignant melanomas by anti-PCNA/cyclin monoclonal antibody. Histopathology 1991;18:221-7.
16 Kanter L, Blegen $\mathrm{H}$, Weide J, et al. Utility of a proliferation marker in distinguishing between benign naevocellular naevi and naevocellular naevuslike lesions with malignant properties. Melanoma Res 1995;5:345-50.

17 Rudolph P, Schubert C, Tamm S, et al. Telomerase activity in melanocytic lesions: a potential marker of tumor biology. Am J Pathol 2000;156:1425-32.

18 Hussein MR, Sun M, Tuthill RJ, et al. Comprehensive analysis of 112 melanocytic skin lesions demonstrates microsatellite instability in melanomas and dysplastic nevi, but not in benign nevi. J Cutan Pathol 2001;28:343-50.

19 Birindelli S, Tragni G, Bartoli C, et al. Detection of microsatellite alterations in the spectrum of melanocytic nevi in patients with or without individual or family history of melanoma. Int J Cancer 2000;86:255-61.

20 Park WS, Vortmeyer AO, Pack S, et al. Allelic deletion at chromosome 9p21 (p16) and 17p13 (p53) in microdissected sporadic dysplastic nevus. Hum Pathol 1998;29:127-30.

21 Aaltonen LA, Peltomaki P, Leach FS, et al. Clues to the pathogenesis of familial colorectal cancer [see comments]. Science 1993;260:812-16.

22 Boni R, Zhuang Z, Albuquerque A, et al. Loss of heterozygosity detected on $1 p$ and $9 \mathrm{q}$ in microdissected atypical nevi [letter]. Arch Dermatol 1998; 134:882-3.

23 Lee JY, Dong SM, Shin MS, et al. Genetic alterations of pl6INK4a and p53 genes in sporadic dysplastic nevus. Biochem Biophys Res Commun 1997;237:667-72.

24 Beitner H, Nakatani T, Hedblad MA. A transmission electron microscopical study of dysplastic naevi. Acta Derm Venereol 1990;70:41 1-16.

25 Newton JA, Bataille V, Griffiths K, et al. How common is the atypical mole syndrome phenotype in apparently sporadic melanoma? J Am Acad Dermatol 1993;29:989-96.

26 Halpern AC, Guerry Dt, Elder DE, et al. Dysplastic nevi as risk markers of sporadic (nonfamilial) melanoma. A case-control study. Arch Dermatol 1991;127:995-9

27 Marghoob AA, Kopf AW, Rigel DS, et al. Risk of cutaneous malignant melanoma in patients with 'classic' atypical-mole syndrome. A case-control study. Arch Dermatol 1994;130:993-8.

28 Bataille V, Bishop JA, Sasieni P, et al. Risk of cutaneous melanoma in relation to the numbers, types and sites of naevi: a case-control study. $\mathrm{Br} J$ Cancer 1996;73:1605-11

29 Weinstock MA, Brodsky GL. Bias in the assessment of family history of melanoma and its association with dysplastic nevi in a case-control study. J Clin Epidemiol 1998;51:1299-303.

30 Carey WP Jr, Thompson CJ, Synnestvedt M, et al. Dysplastic nevi as a melanoma risk factor in patients with familial melanoma. Cancer 1994;74:3118-25.

31 Rhodes AR, Harrist TJ, Day CL, et al. Dysplastic melanocytic nevi in histologic association with 234 primary cutaneous melanomas. J Am Acad Dermatol 1983;9:563-74.

32 Tucker MA, Halpern A, Holly EA, et al. Clinically recognized dysplastic nevi. A central risk factor for cutaneous melanoma [see comments]. JAMA 1997;277:1439-44

33 Bataille V, Grulich A, Sasieni P, et al. The association between naevi and melanoma in populations with different levels of sun exposure: a joint casecontrol study of melanoma in the UK and Australia. $\mathrm{Br} J$ Cancer 1998:77:505-10.

34 Howell JN, Greene MH, Corner RC, et al. Fibroblasts from patients with hereditary cutaneous malignant melanoma are abnormally sensitive to the mutagenic effect of simulated sunlight and 4-nitroquinoline 1-oxide. Proc Natl Acad Sci U S A 1984;81:1179-83.

35 Smith PJ, Greene MH, Devlin DA, et al. Abnormal sensitivity to UV-radiation in cultured skin fibroblasts from patients with hereditary cutaneous malignant melanoma and dysplastic nevus syndrome. Int J Cancer 1982;30:39-45.

36 Moriwaki SI, Tarone RE, Tucker MA, et al. Hypermutability of UV-treated plasmids in dysplastic nevus/familial melanoma cell lines. Cancer Res 1997; 57:4637-41.

37 Caporaso N, Greene MH, Tsai S, et al. Cytogenetics in hereditary malignant melanoma and dysplastic nevus syndrome: is dysplastic nevus syndrome a chromosome instability disorder? Cancer Genet Cytogenet 1987;24:299-314.

38 Clark WH Jr, Elder DE, Van Horn M. The biologic forms of malignant melanoma. Hum Pathol 1986;17:443-50.

39 Rhodes AR, Seki Y, Fitzpatrick TB, et al. Melanosomal alterations in dysplastic melanocytic nevi. A quantitative, ultrastructural investigation. Cancer 1988;61:358-69.

40 Cristofolini M, Boi S, Girlando S, et al. p53 protein expression in nevi and melanomas. Arch Dermatol 1993; 129:739-43.

41 Bergman W, Ruiter DJ, Scheffer E, et al. Melanocytic atypia in dysplastic nevi. Immunohistochemical and cytophotometrical analysis. Cancer 1988;61:1660-6.

42 Newton JA, Camplejohn RS, McGibbon DH. The flow cytometry of melanocytic skin lesions. Br J Cancer 1988;58:606-9

43 Bale SJ, Chakravarti A, Greene MH. Cutaneous malignant melanoma and familial dysplastic nevi: evidence for autosomal dominance and pleiotropy. Am J Hum Genet 1986;38:188-96.

44 Clark WH Jr, Elder DE, Guerry Dt, et al. A study of tumor progression: the precursor lesions of superficial spreading and nodular melanoma. Hum Pathol $1984 ; 15: 1147-65$.

45 Naeyaert JM, Brochez L. Clinical practice. Dysplastic nevi. N Engl J Med 2003;349:2233-40

46 Ackerman AB, Massi D, Nielsen TA. Dysplastic nevus: atypical mole or typical myth. Philadelphia: Ardor Scribendi Ltd, 1999.

47 Ackerman $A B$, Elder DE. An exchange of ideas about dysplastic nevi and malignant melanomas. Am J Dermatopathol 1985;7:99-105. 